\title{
Calculation of Bending Losses in Dielectric Waveguides Using Eigenmode Expansion and Perfectly Matched Layers
}

\author{
Peter Bienstman, Student Member, IEEE, E. Six, M. Roelens, M. Vanwolleghem, Student Member, IEEE, and \\ Roel Baets, Senior Member, IEEE
}

\begin{abstract}
In this letter, we present a different approach to accurately calculate the bending losses in curved dielectric waveguides. It is based on the well-known conformal transformation of the index profile and on vectorial eigenmode expansion, but this time with perfectly matched layer (PML) boundary conditions to accurately model radiation losses. The modal spectrum of these waveguides in the presence of PML is discussed and the method is validated by comparing it to previously published results.
\end{abstract}

Index Terms-Absorbing boundary conditions, bending losses, eigenmode expansion, waveguide bends, waveguide theory.

\section{INTRODUCTION}

B ENDS in dielectric waveguides are an important building block for nearly all types of photonic integrated circuits (PICs). Therefore, it is necessary to be able to design and simulate these bends in an accurate and speedy manner. More specifically, the bending radiation losses have to be modeled correctly, as these are an important parameter for the performance of the device.

Most techniques modeling bends in dielectric waveguides use the so-called conformal transformation of the index profile, where the piecewise constant index profile in a two-dimensional (2-D) circular geometry $r$ is transformed to an exponential field profile in a straight geometry $u[1]$

$$
\begin{aligned}
u & =R \ln \frac{r}{R} \\
n_{t} & =n \exp \frac{u}{R}
\end{aligned}
$$

For the analysis of three-dimensional (3-D) bends, the index profile can first be reduced to 2-D by applying the effective index method prior to the conformal transformation.

The straight waveguide with the transformed index profile can be analyzed with a number of techniques, ranging from beam propagation methods (BPM) [2] to eigenmode expansion

\footnotetext{
Manuscript received July 13, 2001; revised October 19, 2001. P. Bienstman was supported by the Flemish Fund for Scientific Research (FWO-Vlaanderen) through a doctoral fellowship. M. Vanwolleghem was supported by the Institute for the Promotion of Innovation Through Science and Technology (IWT).

The authors are with the Department of Information Technology, Ghent University, B-9000 Gent, Belgium (e-mail Peter.Bienstman@ rug.ac.be).

Publisher Item Identifier S 1041-1135(02)00257-4.
}

methods [3]. As BPM is intrinsically an approximate method, eigenmode techniques seem more advantageous. However, in classical eigenmode methods, the structure needs to be enclosed between two perfectly conducting metal walls in order to discretize the mode spectrum. This causes parasitic reflections, because the radiation lost in the bend is totally reflected at the metal walls and can disturb the simulation results. In fact, these parasitic reflections make it completely impossible to determine the radiation losses of the waveguide modes, since they will all have real propagation constants because of energy conservation in lossless media enclosed by perfect reflectors.

Another approach to calculate these radiation losses is to eliminate the metal walls and estimate the losses from the leaky modes of the completely open structure. These leaky modes are complex improper solutions to the open dispersion relation because their fields profiles increase exponentially toward infinity in the cladding [4]. Although this technique is suited to determine the loss of individual modes, it is well-known that leaky modes do not form a complete set and can, therefore, generally not be used to describe an arbitrary field. This means that leaky mode expansion is not suited to study, e.g., the minimization of the transition loss between a straight and a curved waveguide, because the scattering at the interface between these two sections will give rise to a field that contains more components than a single leaky mode.

In this letter, we present a different eigenmode approach that does not have these disadvantages. We keep the structure enclosed between two metal walls, but we clad these with a perfectly matched layer (PML), which can absorb incident radiation without any additional parasitic reflections, regardless of wavelength, incidence angle, or polarization [5]. This allows us to accurately model radiation losses, while at the same time keeping a discrete set of eigenmodes.

The rest of this letter is organized as follows. In Section II, we will briefly review the eigenmode expansion method combined with PML boundary conditions. Section III will focus on the influence of PML on the modal spectrum of waveguide bends. Simulation results on the radiation losses of the fundamental mode will be given in Section IV. In Section V, we will use the technique to model the more complicated problem of determining the optimal offset at the transition between a straight and a curved waveguide. Finally, Section VI will summarize and conclude this letter. 


\section{EIGENMODE EXPANSION WITH PML}

For the sake of completeness, we will briefly reiterate the main points of the PML eigenmode expansion method, which we already described in more detail in [6].

PML is included in the model by making use of the complex coordinate stretching technique [7], which states that a PML can be described by a layer with a real refractive index, but with a complex thickness. The imaginary part of this complex thickness provides the reflectionless absorption of the incident fields.

The rest of the eigenmode expansion model follows naturally; the dispersion relation is solved for the propagation constants of the eigenmodes in each longitudinally invariant section of the device under study. From their field profiles and the overlap integrals of these modes, the scattering matrix describing the interface between two different sections is constructed using the mode-matching technique. Finally, the scattering matrix of the entire sequence of sections making up the device is calculated.

Because eigenmode expansion does not rely on spatial discretization, it typically requires far fewer unknowns and far less computational effort than other models.

\section{Modal Spectrum of CuRved Waveguides IN THE PRESENCE OF PML}

In this section, we will study the propagation constants of a curved waveguide with the following properties: core index 3.24 , cladding index 3.17 , width $w=3 \mu \mathrm{m}$, bending radius $R=50 \mu \mathrm{m}$. After conformal transformation, we obtain an exponential field profile, which we approximate with a sufficiently fine staircase profile (50 steps). The total thickness of the low index cladding is $10 \mu \mathrm{m}$, that of the high index is $17 \mu \mathrm{m}$. The PML is incorporated by giving the outermost staircase step in both claddings an imaginary thickness of $-0.5 \mathrm{j} \mu \mathrm{m}$, which turned out to be sufficient for the calculated radiation losses to be independent on a further increase in PML absorption. The operation wavelength is $1.55 \mu \mathrm{m}$, and we study the TM modes.

In the absence of PML, i.e., with zero imaginary cladding thickness, all modes lie on the coordinate axes, which as we already said makes it impossible to determine the radiation loss of the bend modes. Moreover, apart from the modes guided by the bend, there are also modes guided only by the outer highindex region of the cladding, which has a much higher refractive index than the waveguide core.

With the introduction of PML, the modal spectrum is completely different, as can be seen in Fig. 1, showing the distribution of hundred eigenmodes for the waveguide structure described in the beginning of this section. The fundamental mode is easily identifiable as the mode marked "A" in Fig. 1, as it lies closest to the positive real axes (top edge of the figure) and closest to the index of the core. Other branches of modes exist with higher losses, e.g., the branches containing mode B or C.

Fig. 2 shows the magnitude of the magnetic field for modes $\mathrm{A}, \mathrm{B}$, and $\mathrm{C}$, together with the transformed index profile. It is clearly visible that mode $\mathrm{A}$ is guided by the waveguide, but leaks some power to the outside of the bend (right side of the figure), where it has a plane-wave like component indicative of radial radiation loss. The modes B and C are not guided by the bend, but only by the high index occurring in the outer cladding.

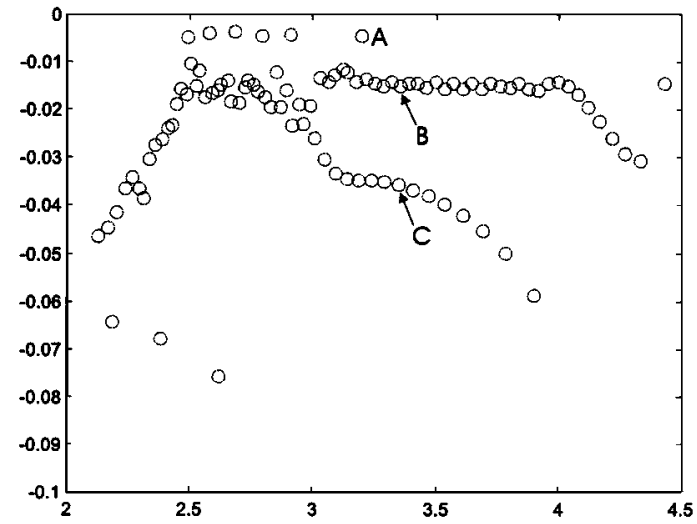

Fig. 1. Distribution of propagation constants in the $n_{\text {eff }}$-plane. Horizontal axis is the real $n_{\mathrm{cff}}$-axis and vertical axis the imaginary $n_{\mathrm{cff}}$-axis.

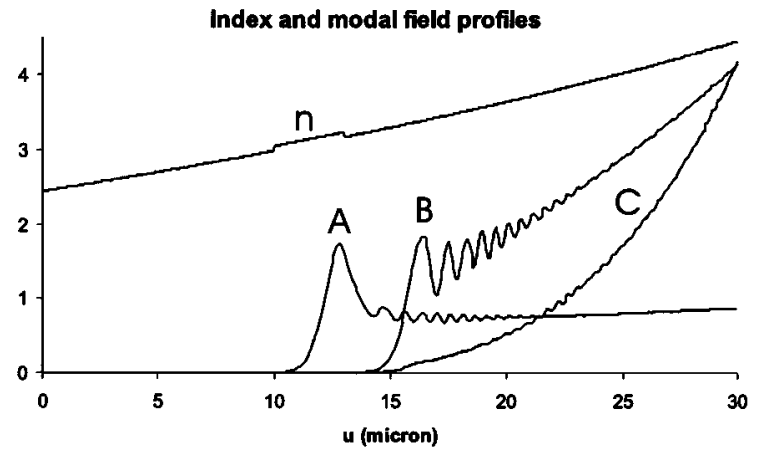

Fig. 2. Transformed index profile and magnitude of the magnetic fields of the modes marked in Fig. 1.

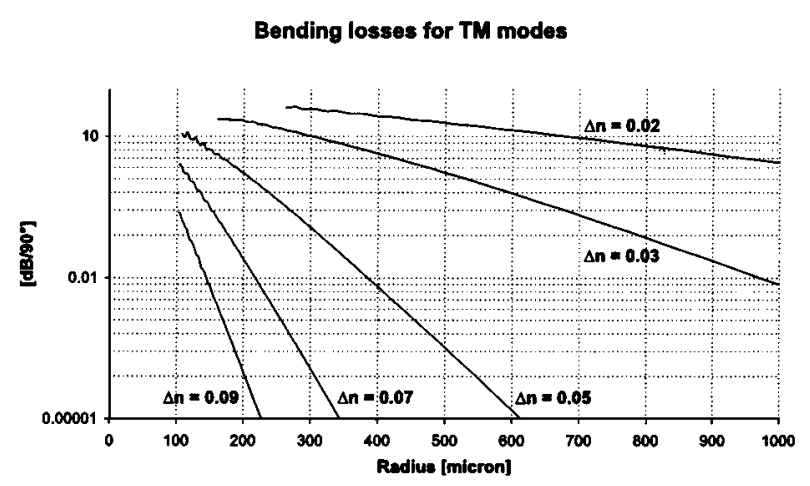

Fig. 3. Radiation loss per $90^{\circ}$ as a function of bend radius and index contrast

\section{RADIATION LOSSES}

Fig. 3 shows the calculated radiation losses of the same waveguide geometry as used previously, as a function of bending radius and refractive index contrast (as obtained by varying the cladding index). The radiation loss was calculated from the imaginary part of the effective index. The results follow the well-known trends that losses increase for shorter bending radius and for lower index contrast, both because of lack of confinement. There is a very good quantitative agreement with results obtained from a leaky mode analysis [8]. This is not surprising, because for high PML absorption, some modes in the spectrum converge to the leaky modes of the corresponding structure, as we already showed in [9]. 
Optimal offset $(\mu \mathrm{m})$

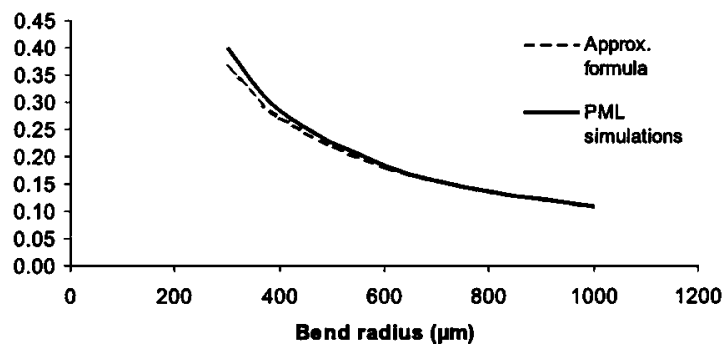

Fig. 4. Optimal lateral offset between a straight and a curved waveguide to maximize transmission at their interface.

\section{OPTIMAL OFFSET}

Because of the mismatch between the modal profiles of the straight and the curved waveguide, scattering will occur when light propagates across the interface between a straight and a curved waveguide. As the mode in the curved section is located closer to the outer waveguide edge (see Fig. 2), it makes sense to laterally offset the straight input waveguide in order to get a better match between the field profiles. By numerically varying this offset, we can determine its optimal value where the transmission of the fundamental mode across the interface is maximal. The results are shown in Fig. 4, for the same geometry as from Section III. These results are also compared to an approximate formula derived in [10]

$$
d=\frac{\pi^{2} n_{\mathrm{eff}}^{2} w^{4}}{\lambda^{2} R} .
$$

The agreement is very good for large bend radii. For smaller bends, the results start to differ slightly, because the radiation losses become more prominent and these losses are more accurately captured by the rigorous PML eigenmode expansion tool.

\section{CONCLUSION}

We presented an alternative approach to model conformally transformed curved waveguides. This approach is based on vectorial eigenmode expansion combined with PML absorbing boundary conditions, such that radiation losses can be accurately determined. We investigated the modal spectrum of these waveguides in the presence of PML and verified our model by comparing it to previously published results in literature. We believe the proposed method is more accurate than both BPM (because BPM introduces approximations) and classical eigenmode methods (because hard walls are replaced with PML boundaries).

\section{ACKNOWLEDGMENT}

Parts of this work were performed in the framework of the Belgian DWTC under project IUAP IV 13.

\section{REFERENCES}

[1] M. Heiblum and J. H. Harris, "Analysis of curved optical waveguides by conformal transformation," IEEE J. Quantum Electron., vol. 11, pp. 75-83, Apr. 1972.

[2] R. Scarmozzino, A. Gopinath, R. Pregla, and S. Helfert, "Numerical techniques for modeling guided-wave photonic devices," IEEE J. Select. Topics Quantum Electron., vol. 6, pp. 150-162, Jan./Feb. 2000.

[3] G. Sztefka and H. P. Nolting, "Bidirectional eigenmode propagation for large refractive index steps," IEEE Photon. Technol. Lett., vol. 5, pp. 554-557, May 1993.

[4] A. W. Snyder and J. D. Love, Optical Waveguide Theory. London, U.K.: Chapman and Hall, 1983.

[5] J.-P. Bérenger, "A perfectly matched layer for the absorption of electromagnetic waves," J. Comput. Phys., vol. 114, no. 1, pp. 185-200, 1994.

[6] P. Bienstman and R. Baets, "Optical modeling of photonic crystals and vcsels using eigenmode expansion and perfectly matched layers," Opt. Quantum Electron., submitted for publication.

[7] W. C. Chew, J. M. Jin, and E. Michielssen, "Complex coordinate stretching as a generalized absorbing boundary condition," Microwave Opt. Technol. Lett., vol. 15, no. 6, pp. 363-369, 1997.

[8] D. Van Thourhout, "Multiwavelength Lasers for WDM-Networks: Theory, Design and Realization by Hybrid Integration," Ph.D. dissertation, Ghent Univ., 2000.

[9] P. Bienstman and R. Baets, "Advanced boundary conditions for eigenmode expansion models," Opt. Quantum Electron., submitted for publication.

[10] E. C. M. Pennings, "Bends in Optical Ridge Waveguides," Ph.D. dissertation, Delft Univ. of Technol., 1990. 